\title{
Explant culture of sarcoma patients' tissue
}

\author{
Roman Muff ${ }^{1}$, Sander M Botter ${ }^{1}$, Knut Husmann ${ }^{1}$, Joelle Tchinda², Philomina Selvam ${ }^{1}$, Franziska Seeli-Maduz ${ }^{1}$ and \\ Bruno Fuchs ${ }^{1}$
}

Human sarcomas comprise a heterogeneous group of rare tumors that affect soft tissues and bone. Due to the scarcity and heterogeneity of these diseases, patient-derived cells that can be used for preclinical research are limited. In this study, we investigated whether the tissue explant technique can be used to obtain sarcoma cell lines from fresh as well as viable frozen tissue obtained from 8 out of 12 soft tissue and 9 out of 13 bone tumor entities as defined by the World Health Organization. The success rate, defined as the percent of samples that yielded sufficient numbers of outgrowing cells to be frozen, and the time to freeze were determined for a total of 734 sarcoma tissue specimens. In 552 cases (75\%) enough cells were obtained to be frozen at early passage. Success rates were higher in bone tumors (82\%) compared with soft tissue tumors (68\%), and the mean time to freezing was lower in bone tumors (65 days) compared with soft tissue tumors ( 84 days). Overall, from $40 \%$ of the tissues cells could be frozen at early passage within $<2$ month after tissue removal. Comparable results as with fresh tissue were obtained after explant of viable frozen patient-derived material. In a selected number of bone and soft tissue sarcoma entities, conventional karyotyping and/or FISH (fluorescence in situ hybridization) analysis revealed a high amount ( $>60 \%$ ) of abnormal cells in $41 \%$ of analyzed samples, especially in bone sarcomas (osteosarcoma and Ewing sarcoma). In conclusion, the explant technique is well suited to establish patientderived cell lines for a large majority of bone and soft tissue sarcoma entities with adequate speed. This procedure thus opens the possibility for molecular analysis and drug testing for therapeutic decision making even during patient treatment.

Laboratory Investigation (2016) 96, 752-762; doi:10.1038/labinvest.2016.49; published online 25 April 2016

Sarcomas are rare, accounting for $\sim 1 \%$ of all tumor types. Nevertheless, the heterogeneity within sarcomas is very large; currently, soft tissue and bone sarcomas are classified into 25 main categories by the World Health Organization (WHO), ${ }^{1}$ and in total $>180$ different sarcoma subtypes are distinguished. Because of this large diversity, patient cohorts are generally small and the available amount of patient tumor material is scarce. Hence, progress in sarcoma research is relatively slow, and bona fide breakthroughs (eg, the introduction of chemotherapy in the 1970s, or more recently, Imatinib in c-kit positive gastrointestinal tumors) are rare. As a result, patient survival of many types of sarcomas has not been significantly improved over the last few decades. ${ }^{2}$

Present-day preclinical sarcoma research, like many other cancer research areas, is largely relying on commercially available 'standard' cell lines, many of which who were developed in the 1950s and 1960s with largely unknown patients history. Recent genetic analyses have shown that with continued passaging of these cells, new genetic changes are introduced as a consequence of the inherent genetic or phenotypic instability of these cell lines., ${ }^{3,4}$ Therefore, it is possible that some characteristics of these cells no longer reflect the cellular processes occurring early in the patient. Consequently, it is important to focus more on primary, patient-derived material with known patient's history in addition to these commercially available standard cell lines.

So far, cell lines have been obtained from sarcomas mainly by two methods; seeding material from pleural effusions ${ }^{5-12}$ or seeding of cells after enzymatic digestion of tumor material. ${ }^{13-19}$ As we assumed it to be difficult to optimize enzymatic digestion protocols for each single tissue type affected by sarcoma and because pleural effusion is mainly performed in patients with end-stage lung metastases only, we explored here the potential to obtain patient-derived cell lines from sarcoma patients using the explant technique. Notably, apart from incidental case reports, ${ }^{20-25}$ this technique has so

${ }^{1}$ Laboratory for Orthopedic Research, Department of Orthopedics, University of Zurich, Zurich, Switzerland and ${ }^{2}$ Oncology Laboratory, University Children's Hospital Zurich, Zurich, Switzerland

Correspondence: Professor B Fuchs, MD, PhD, University of Zurich, Balgrist University Hospital, Laboratory for Orthopedic Research, Forchstrasse 340, Zurich 8008, Switzerland.

E-mail: bfuchs@research.balgrist.ch

Received 2 October 2015; revised 8 March 2016; accepted 3 March 2016 
far not been investigated thoroughly for sarcomas in general or for specific sarcoma entities. Here, we show that this technique yields enriched cancer cells at early passages in $41 \%$ of explanted specimens. These patient-derived cell lines can be used for in vitro studies as drug testing for personalized medicine, but also for establishing additional animal models by orthotopic injection into immunocompromised mice, or for linkage studies when the clinical history of the samples is known.

\section{MATERIALS AND METHODS \\ Explant Culture}

The collection and use of human tissue samples has been approved by the local ethic committees (KEK-10/2007). Samples were processed in a level 2 laboratory according to the regulations of the Swiss authorities.

Samples (711) were from patients in Switzerland with $>95 \%$ collected at Balgrist University Hospital, and were explanted immediately after removal without prior freezing. Twenty-one osteosarcoma and two chondrosarcoma samples were collected at The Denver Clinic for Extremities at Risk (Denver, CO, USA), frozen in DMEM containing 10\% DMSO and shipped on dry ice to Balgrist University Hospital. Upon arrival, samples were thawed quickly and cultured as described below.

Shortly, tissue obtained from diagnostic biopsies (BX) or resections (RX) from primary tumors, recurrences or metastases were placed in tissue culture medium (DMEM, $4.5 \mathrm{~g} / \mathrm{l}$ glucose:F12 (1:1); 10\% FCS; 1\% antibiotic-antimycotic (Gibco, Basel, Switzerland)). Upon arrival in the lab, usually $<1 \mathrm{~h}$ after excision, the material was cut into smaller pieces of about $3 \times 3 \times 3 \mathrm{~mm}$, transferred to $25 \mathrm{~cm}^{2}$ tissue culture flasks and cultured at $37^{\circ} \mathrm{C}, 5 \% \mathrm{CO}_{2}$. Outgrowth of cells was monitored weekly. When cells were distributed equally within the flask, the cells were fed weekly until the flask became almost confluent. When spot-like outgrowth was observed, a trypsinization step $(0.05 \%$ trypsin and $0.02 \%$ EDTA in PBS) was performed and cells were seeded back in the same flask to allow an even distribution of the outgrown cells. After the $25 \mathrm{~cm}^{2}$ flask reached near confluence it was split (passage 1) by trypsinization in four (osteosarcoma), three (chondrosarcoma and Ewing's sarcoma) or two (all other entities) $75 \mathrm{~cm}^{2}$ flasks. After the passage 1 cells reached confluence, the cells were detached by trypsinization and frozen in tissue culture medium $(90 \%)$ and DMSO (10\%) at $37.5 \mathrm{~cm}^{2}$ equivalents per ampoule.

Tumor samples from which outgrown cells were successfully isolated were classified as 'growing', all others as 'nongrowing'. Of these non-growing samples, $>80 \%$ showed outgrowth of viable cells, but thereafter did not proliferate enough to subsequently be frozen. Success rates were calculated as percentage of samples containing growing cells compared with the total amount of samples within each entity. The time period $(\Delta$; days) between explant and freezing of the outgrowing cells (time to freezing) was used as a parameter to compare differences in proliferation speed between individual sarcoma entities. This time period is influenced by several components; the amount, size distribution (total surface area) and viability of the explanted material, the ability of the cells to outgrow, and finally the proliferation rate of the outgrown cells on plastic dishes.

\section{Karyotyping by GTG Banding}

Four to five T25 cell culture flasks with $5 \mathrm{ml}$ cell culture medium at $\sim 60 \%$ confluence were obtained for each sample. Cultures were incubated with $25 \mu \mathrm{l}$ colcemid (KaryoMAX Colcemid Solution, $10 \mu \mathrm{g} / \mu \mathrm{l}$; Life Technologies, Zug, Switzerland) at $37^{\circ} \mathrm{C}$ and $5 \% \mathrm{CO}_{2}$ for $6,24,48,72$ and $96 \mathrm{~h}$ before harvest. Chromosome preparation was done using standard techniques. ${ }^{26}$ Briefly, the adherent cells were detached using incubation with trypsin, followed by hypotonic treatment, fixation with methanol-acetic acid, slide preparation and GTG banding. Metaphases were searched and captured using an automated microscope equipped with a scanning software (Metafer, MetaSystems GmbH, Altlussheim, Germany). At least 10 metaphases were analyzed using the Metasystems IKAROS software and the karyotypes were reported according to the latest ISCN. ${ }^{27}$ Normal samples ( $0 \%$ abnormality) were distinguished from samples with low $(0-20 \%)$, intermediate (20-60\%), and high (60-100\%) presence of abnormal metaphases.

\section{FISH Analysis}

Fluorescence in-situ hybridization (FISH) was performed according to the protocol of the manufacturer using the MDM2 Amplification probe and the EWSR1 Breakapart probe (Cytocell, Cambridge, UK). Briefly, cytospins with patient cells and $2 \mu \mathrm{l}$ probes were co-denatured at $75^{\circ} \mathrm{C}$ and hybridized overnight at $37^{\circ} \mathrm{C}$. After two post-hybridization washes, fluorescent signals were viewed and analyzed with an Axio Imager Z.2 fluorescence microscope equipped with filters for DAPI, FITC, TRITC, and DEAC (Carl Zeiss AG, Feldbach, Switzerland). Images were captured with a Cool Cube 1 camera and the ISIS software (MetaSystems $\mathrm{GmbH}$, Altlussheim, Germany). Two-hundred nuclei were analyzed for each hybridization.

\section{Immunocytochemistry}

Cells were seeded on gelatin-coated $(0.1 \%)$ glass slides in 24-well plates at a density of 2500-10000 cells per well. Cells were allowed to attach and spread on the slides and were then fixed with $4 \%$ formalin for $5 \mathrm{~min}$ at room temperature. Cells were washed with PBS and remaining formalin was neutralized by incubation with cell culture medium containing 10\% FCS for $30 \mathrm{~min}$ at room temperature. Cells were washed with PBS and incubated with anti-CD99 antibody (Abcam, Cambridge, UK; clone 12E7; ab8855; 1:100; $2 \mu \mathrm{g} / \mathrm{ml}$ ) or isotype control (mouse $\mathrm{IgG}_{1}$, kappa (Becton Dickinson, San Jose, CA, USA); \#557273; 1:250; $2 \mu \mathrm{g} / \mathrm{ml}$ ) in PBS, $1 \%$ 
bovine serum albumin for $2 \mathrm{~h}$ at room temperature. Cells were washed three times with PBS and incubated with Alexa546 anti-mouse (Invitrogen, Carlsbad, CA, USA; A11030; 1:400) and Hoechst dye (Sigma, St. Louis, MO, USA; H3570; $5 \mu \mathrm{g} / \mathrm{ml}$ ) in PBS, $1 \%$ bovine serum albumin for $30 \mathrm{~min}$ at room temperature. Cells were washed three times with PBS and slides mounted with Immu-mount (Thermo Scientific, Waltham, MA, USA). Slides were viewed with the 10x objective and respective fluorescence filter blocks using an Axiocam MRm camera attached to a Zeiss Observer.Z1 microscope (Carl Zeiss AG, Feldbach, Switzerland). For estimating the percentage of CD99-positive cells, cells were detached with trypsin/EDTA and staining was performed in suspension under the conditions described above. Images were analyzed with ImageJ (http://imagej.nih. gov/ij/).

\section{Statistical Analysis}

Success rates were compared with $\chi^{2}$ test if $n$ were $>5$, and otherwise with two-tailed Fisher exact probability test using VassarStats (http://vassarstats.net/). Differences between means were compared with paired or unpaired $t$-test using PRISM 5.

\section{RESULTS}

\section{Sample Description}

Between June 2005 and November 2014 (9.5 years) a total of 734 tissue samples from sarcoma patients were cultured by the explant technique as described in Materials and Methods section. Table 1 divides these samples into the different sarcoma types according to the 2013 WHO sarcoma classification. Samples (552) were classified as 'growing', whereas 182 samples were viable with outgrowing cells, but stopped proliferation and did not expand sufficiently to be considered for freezing and storage (ie, 'non-growing'). Of all, 323 samples (44\%) were from BX, $382(52 \%)$ from surgical RX, $16(2 \%)$ from metastases (M) and $13(2 \%)$ from recurrences $(\mathrm{RC})$.

\section{Success Rates and Times to Freezing in Soft Tissue vs Bone Sarcomas}

We first compared the overall success rates, defined as percent of samples that yielded enough cells to be frozen, between bone and soft tissue sarcoma. In bone sarcomas, 317 out of 388 samples (success rate: $82 \%$ ) were classified as 'growing', which was significantly higher $(P<0.0001)$ compared with 235 out of 346 soft tissue sarcomas (success rate 68\%: Table 2). The mean success rate of all entities was $81 \%$ with a $95 \%$ confidence interval (CI) of $73-88 \%$. In soft tissue sarcoma three $(38 \%)$ entities (adipocytic and nerve sheath tumors and undifferentiated/unclassified sarcomas) had lower success rates than the lower CI, and two (25\%) entities (so-called fibrohistiocytic and pericytic (perivascular) tumors) had higher success rates than the upper $\mathrm{CI}$ of the mean success rate. On the other hand, in bone sarcoma one (11\%)
Table 1 WHO classification of sarcomas

\author{
Soft tissue tumors \\ Adipocytic tumors \\ Fibroblastic/myofibroblastic tumors \\ So-called fibrohistiocytic tumors \\ Pericytic (perivascular tumors) \\ Vascular tumors \\ Nerve sheath tumors \\ Tumors of uncertain differentiation \\ Undifferentiated/unclassified sarcomas \\ Bone tumors \\ Chondrogenic tumors \\ Osteogenic tumors \\ Ewing sarcoma \\ Haematopoietic neoplasms \\ Osteoclastic giant cell-rich tumors \\ Myogenic, lipogenic, and epithelial tumors \\ Tumors of undefined neoplastic nature \\ Undifferentiated high-grade pleomorphic sarcoma \\ Tumor syndromes
}

entity (haemotopoietic neoplasms) had lower success rate than the lower CI, and four (44\%) entities (osteogenic, osteoclastic giant cell-rich, myogenic, lipogenic, and epithelial tumors, and undifferentiated high-grade pleomorphic sarcoma) had higher success rates than the upper CI of the mean success rate.

The mean time to freezing $(\Delta)$ of all entities was 74 days with a CI of 64-83 days (Table 2). The mean time to freezing of the bone sarcomas (65 days) was significantly $(P<0.03)$ lower than that of soft tissue sarcomas of 84 days, respectively. In soft tissue sarcoma $4(50 \%)$ entities (adipocytic and fibroblastic/myofibroblastic tumors and undifferentiated/ unclassified sarcomas) had higher times to freezing then the upper CI of the mean time to freezing and none $(0 \%)$ had lower times to freezing than the lower $\mathrm{CI}$ of the mean time to freezing. On the other hand, in bone sarcoma four entities (osteogenic tumors, Ewing sarcoma, myogenic, lipogenic, and epithelial tumors and undifferentiated high-grade pleomorphic sarcoma) showed times to freezing below the lower CI and only one (11\%) entity (tumor syndromes) had higher time to freezing than the upper CI of the mean time to freezing.

In conclusion, the explant technique is suitable to obtain cells from patients' tissue for most of the sarcoma entities investigated, and was particularly applicable in samples from bone tumors. 


\begin{tabular}{|c|c|c|c|c|c|c|}
\hline & \multicolumn{3}{|c|}{ Number of samples } & \multirow{2}{*}{$\begin{array}{c}\text { Success } \\
\%\end{array}$} & \multirow[t]{2}{*}{$\Delta$ Mean } & \multirow[t]{2}{*}{$\Delta$ Range } \\
\hline & $a$ & $b$ & $c$ & & & \\
\hline \multicolumn{7}{|l|}{ Soft tissue tumors } \\
\hline Adipocytic tumors & 74 & 56 & 130 & 57 & 114 & $100-128$ \\
\hline Fibroblastic/myofibroblastic tumors & 44 & 15 & 59 & 75 & 92 & $78-106$ \\
\hline So-called fibrohistiocytic tumors & 30 & 2 & 32 & 94 & 72 & $62-82$ \\
\hline Pericytic (perivascular tumors) & 6 & 0 & 6 & 100 & 72 & $56-88$ \\
\hline Vascular tumors & 9 & 3 & 12 & 75 & 67 & $53-81$ \\
\hline Nerve sheath tumors & 22 & 12 & 34 & 65 & 93 & 79-107 \\
\hline Tumors of uncertain differentiation & 32 & 5 & 37 & 86 & 70 & $59-82$ \\
\hline Undifferentiated/unclassified sarcomas & 18 & 18 & 36 & 50 & 89 & $62-116$ \\
\hline Total & 235 & 111 & 346 & & & \\
\hline \multicolumn{7}{|l|}{ Bone tumors } \\
\hline Chondrogenic tumors & 88 & 29 & 117 & 75 & 68 & $60-76$ \\
\hline Osteogenic tumors & 96 & 9 & 105 & 91 & 61 & $52-70$ \\
\hline Ewing sarcoma & 19 & 3 & 22 & 86 & 58 & $39-77$ \\
\hline Haematopoietic neoplasms & 21 & 11 & 32 & 66 & 75 & $61-90$ \\
\hline Osteoclastic giant cell-rich tumors & 22 & 1 & 23 & 96 & 81 & $62-100$ \\
\hline Myogenic, lipogenic, and epithelial tumors & 8 & 0 & 8 & 100 & 46 & $20-72$ \\
\hline Tumors of undefined neoplastic nature & 26 & 7 & 33 & 79 & 64 & $49-79$ \\
\hline Undifferentiated high-grade pleomorphic sarcoma & 6 & 0 & 6 & 100 & 42 & $30-54$ \\
\hline Tumor syndromes & 31 & 11 & 42 & 74 & 88 & $73-103$ \\
\hline Total & 317 & 71 & 388 & & & \\
\hline \multirow[t]{2}{*}{ Total } & 552 & 182 & 734 & & & \\
\hline & $81(73-88)^{\mathrm{a}}$ & & $74(64-83)^{\mathrm{a}}$ & & & \\
\hline
\end{tabular}

Abbreviations: $\Delta$, time to freezing; $a$, number of samples containing growing cells; $b$, number of samples without growing cells; $c$, total number of samples. a Total mean values with $95 \% \mathrm{Cl}$. Values of entities with high success or low times to freezing compared with the $95 \% \mathrm{Cl}$ of all entities are indicated in bold, whereas values of entities with low success or high times to freezing compared with the $95 \% \mathrm{Cl}$ of all entities are indicated in italics.

Entities with $>5$ growing samples are listed. The $\Delta$ mean represents the mean time period between initial culture of the explant and freezing of the cells, with the $\Delta$ range representing the $95 \%$ confidence interval.

\section{Dependency of Success Rates and Times to Freeze on Disease Malignancy}

Some sarcoma entities are further subcategorized into benign, intermediate, or malignant based on the ability of the tumor to only grow locally or build metastases. We determined whether these subcategories could explain the variance in success rate, defined as percent of samples that yielded enough cells to be frozen, and time to freeze. Success rates were independent from malignancy in adipocytic, fibroblastic/myofibroblastic tumors, and osteogenic tumors and in tumors of uncertain differentiation (not shown) irrespective whether benign disease or benign plus intermediate disease was compared with malignant disease. In chondrogenic tumors, malignant chondrosarcoma had a significant
$(P<0.03)$ higher $(85 \%)$ success rate than benign plus intermediate disease $(65 \%)$.

The times to freezing were independent on the malignancy in adipocytic, fibroblastic/myofibroblastic, and osteogenic tumors, in tumors of uncertain differentiation and in undifferentiated/unclassified sarcomas (Figure 1a) when benign disease was compared with malignant disease. In chondrogenic tumors, the time to freezing was significantly lower in chondrosarcoma (53 days) compared with benign disease $(79$ days; $P<0.003)$ and intermediate disease (73 days; $P<0.05$; Figure 1b). A similar trend was observed in osteogenic tumors $(P<0.11)$ and tumors of uncertain differentiation $(P<0.14)$ when benign plus intermediate disease was compared with malignant disease (Figure 1a). 


\begin{tabular}{|c|c|c|c|c|c|}
\hline Tumors & benign & interm. & malign & Pt & Ptt \\
\hline & \multicolumn{3}{|c|}{$\mathrm{n}$} & \multicolumn{2}{|c|}{$\Delta$} \\
\hline Adipocytic & 50 & 2 & 22 & 0.65 & 0.71 \\
\hline $\begin{array}{l}\text { Fibroblastic/ } \\
\text { myofibroblastic }\end{array}$ & 18 & 10 & 16 & 0.69 & 1.00 \\
\hline Vascular & 8 & 0 & 1 & n.a. & n.a. \\
\hline Nerve sheath & 17 & 0 & 5 & 0.42 & n.a. \\
\hline $\begin{array}{l}\text { Of uncertain } \\
\text { differentiation }\end{array}$ & 19 & 3 & 10 & 0.24 & 0.14 \\
\hline Chondrogenic & 33 & 23 & 32 & 0.003 & 0.004 \\
\hline Osteogenic & 3 & 3 & 90 & 0.20 & 0.11 \\
\hline $\begin{array}{l}\text { Osteoclastic } \\
\text { giant cell-rich }\end{array}$ & 0 & 22 & 0 & n.a. & n.a. \\
\hline
\end{tabular}

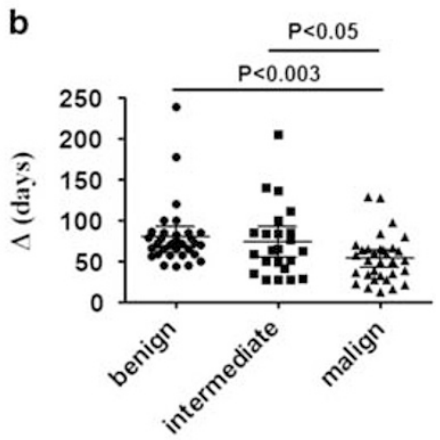

Figure 1 Relation between malignancy and time to freezing. (a) Number ( $n$ ) of samples and $P$ for time to freezing ( $\Delta$ ) values for benign versus malign comparison $(\dagger)$, and benign plus intermediate versus malign comparison ( $\dagger \dagger)$. Values in black indicate significant differences. (b) The distribution of values in chondrogenic tumors with means and 95\% confidence intervals.

In hematopoietic neoplasms of the bone, with malignant disease only, the lowest success of 33\% was obtained with plasma cell myeloma and highest success $(73 \%)$ with primary non-Hodgkin lymphoma.

\section{Dependency of Success Rates and Times to Freeze on the Source of Material}

When all sarcoma subtypes were considered, the overall success rates and time to freezing did not differ between BX- and RXderived material (not shown), however, within specific sarcoma entities significant differences were observed. Success rates were significantly $(P<0.05)$ lower in RX $(48 \%)$ compared with BX $(67 \%)$ in adipocytic tumors (Figure $2 \mathrm{a})$. Conversely, in chondrogenic tumors the success rates were lower $(P<0.02)$ in BX (59\%) compared with RX (83\%).

The time to freezing was significantly $(P<0.05)$ higher in RX $(\Delta=140$ days) when compared to $\mathrm{BX}(\Delta=106$ days $)$ in adipocytic tumors, whereas in chondrogenic tumors the time to freezing was lower $(P<0.02)$ in $\mathrm{RX}$ (62 days) when compared with BX ( 85 days; Figure 2a; distributions shown in Figures 2b and $c$ ). When paired samples (ie, BX and RX of the same patient) were compared, a trend $(P<0.13$ and 0.21 , respectively) towards similar tendencies was observed (Figure 2a). Furthermore, paired analyses gave significant $(P<0.03)$ lower times to freezing in RX compared with $\mathrm{BX}$ in so-called fibrohistiocytic tumors and tumors of uncertain differentiation (Figure 2a; individual sample pairs shown in Figures 2d and e).

\section{Dependency of Success Rates on Freezing Samples Before Explant Culture}

From 23 samples (21 osteosarcoma and 2 chondrosarcoma) obtained frozen from The Denver Clinic for Extremities at
Risk, 20 (87\%) were viable after thawing, with outgrowing cells in the explant culture. The success rate, defined as percent of samples that yielded enough cells to be frozen, was $76 \%$. Biopsy- and resection-derived patient material was also immediately frozen from patients in our hospital, and 20 randomly selected samples (5 nerve sheet, 4 chondrogenic, 4 adipocytic, and 7 other sarcoma entities) were thawed and tested for viability. Of all, 17 (85\%) showed viability also in this cohort. Two of the three frozen samples that failed were resections that also showed no success in direct explants without prior freezing. Therefore, high viability after freezing and thawing was observed independent of the tumor origin.

\section{Characterization of Cells by Karyotyping and FISH}

A tumor in situ consists of a mixture of tumor cells and supporting stromal cells (fibroblasts and endothelial cells). Because the explant technique does not discriminate between the outgrowth of both cell types, the resulting cell cultures may also consist of a mixture of tumor and stromal cells. To estimate the percentage of tumor cells, conventional karyotyping was carried out in samples (both BX and RX) from three sarcoma entities representing the malignant forms of adipocytic and osteogenic tumors and in Ewing sarcoma. Thus, eight osteosarcoma, one Ewing patient and one patient with myxoid liposarcoma were analyzed (Figure 3). Karyotyping was performed after thawing passage 1 cells at passages 2-43 (median 3; 25-75\% percentile 2-8). All seven cell lines karyotyped at passages $\geq 5$ showed a high percentage of abnormal cells, whereas only $21 \%$ (3 of 14) of cell lines karyotyped at passages $<5$ exhibited a high percentage of abnormal cells $(P<0.002)$. All osteosarcoma samples were from conventional osteosarcoma, five having osteoblastic and 
a

\begin{tabular}{|c|c|c|c|c|c|c|}
\hline Tumors & $B X$ & $\mathrm{RX}$ & Success & $\Delta t$ & $\begin{array}{l}\text { Paired } \\
B X / R X\end{array}$ & $\Delta t+$ \\
\hline & n & n & $\mathbf{P}$ & $\mathbf{P}$ & n & $\mathbf{P}$ \\
\hline Adipocytic & 41 & 33 & 0.05 & 0.05 & 10 & 0.13 \\
\hline Fibroblastic/myofibroblastic & 21 & 22 & 0.78 & 0.18 & 4 & 0.92 \\
\hline So-called fibrohistiocytic & 11 & 19 & 1.00 & 0.90 & 4 & 0.03 \\
\hline Pericytic (perivascular) & 2 & 4 & n.a & n.a & 1 & n.a \\
\hline Vascular & 4 & 5 & 0.21 & 0.34 & 0 & n.a. \\
\hline Nerve sheath & 10 & 12 & 0.89 & 0.60 & 2 & n.a. \\
\hline Of uncertain differentiation & 14 & 18 & 0.26 & 0.70 & 7 & 0.03 \\
\hline Undifferentiated/unclassified sarcomas & 9 & 8 & 0.86 & 0.18 & 3 & 0.53 \\
\hline Chondrogenic & 23 & 62 & 0.02 & 0.02 & 9 & 0.21 \\
\hline Osteogenic & 36 & 47 & 1.00 & 0.54 & 21 & 0.56 \\
\hline Ewing sarcoma & 11 & 8 & 0.57 & 0.97 & 5 & 0.34 \\
\hline Haematopoietic neoplasms & 16 & 5 & 1.00 & 0.10 & 0 & n.a. \\
\hline Osteoclastic giant cell-rich & 6 & 12 & 1.00 & 0.25 & 5 & 0.85 \\
\hline Myogenic, lipogenic, and epithelial & 3 & 5 & n.a. & 0.92 & 1 & n.a \\
\hline Of undefined neoplastic nature & 11 & 14 & 0.40 & 0.82 & 0 & n.a. \\
\hline Undifferentiated high-grade pleomorphic & 3 & 3 & n.a. & 0.89 & 2 & n.a. \\
\hline Tumor syndromes & 14 & 15 & 0.49 & 0.32 & 2 & n.a. \\
\hline
\end{tabular}
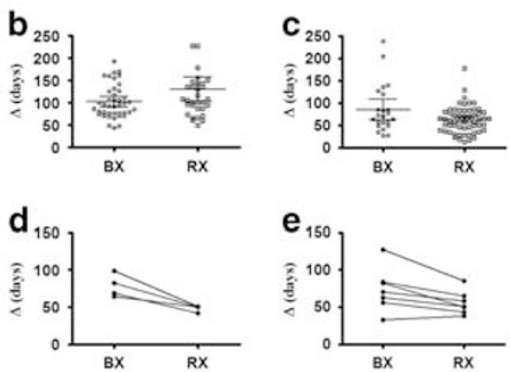

Figure 2 Relation between source of material and success and time to freezing. (a) Time to freezing $(\Delta)$. Number of samples and $P$-values for total comparison ( + ) and paired comparison ( $\dagger+$ ) of biopsies and resections. Values in black indicate significant differences. The distribution of values in adipocytic tumors (b) and chondrogenic tumors (c) with means and 95\% confidence intervals are shown as scatter plots, and paired alignment in so-called fibrohistiocytic tumors (d) and tumors of uncertain differentiation (e).

a

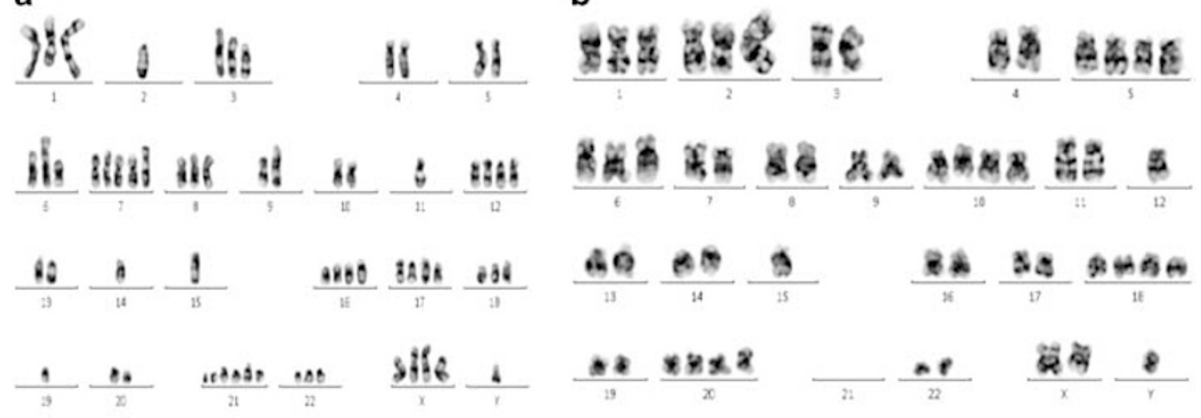

C

\begin{tabular}{|c|c|c|c|c|c|c|c|}
\hline & BX & RX & RC/M & Morph. & $\begin{array}{c}\text { Surv. } \\
\text { (mo) }\end{array}$ & $\begin{array}{c}\text { Resp. } \\
\text { (grade) }\end{array}$ & Resp. \\
\hline OS1 & 0 & 0 & n.p. & ob & LFU & I & yes \\
\hline OS2 & $>60$ & $20-60$ & n.p. & cb & Alive (85) & III & yes \\
\hline OS3 & 0 & 0 & n.p. & cb & Alive (79) & IV & no \\
\hline OS4 & 0 & 0 & n.p. & ob & LFU & IV & no \\
\hline OS5 & 0 & $>60$ & n.p. & ob & DOD (11) & IV & no \\
\hline OS6 & $>60$ & 0 & n.p. & ob & DOD (17) & III & yes \\
\hline OS7 & 0 & $0+$ & $>60(M)$ & $c b$ & DOD (15) & IV & no \\
\hline OS8 & $>60$ & $>60$ & n.p. & ob & DOD (29) & IV-V & no \\
\hline Ewing1 & $>60$ & 0 & $>60(R C)$ & - & DOD (12) & - & - \\
\hline LS1 & $>60$ & n.p. & n.p. & - & Alive (22) & - & - \\
\hline
\end{tabular}

Figure 3 Abnormal karyotypes in osteosarcoma (OS), Ewing sarcoma and myxoid liposarcoma- (LS) derived cells. (a) BX of osteoblastic OS6 and (b) BX of the Ewing patient 1. (c) Percentage of cells with abnormal karyotypes, cell morphology and survival. Values in black indicate high degree of abnormality. + , cells derived from an enzymatic digest, not by the explant technique; RC, recurrence; $M$, metastases; n.p., not present; -, not applicable; ob, osteoblastic; cb, chondroblastic; LFU, lost follow up; DOD, died of disease; grade; Salzer-Kuntschik. ${ }^{40}$ 
three chondroblastic features. High degrees of complex karyotypes were found in 7 of 17 osteosarcoma samples (41\%) derived from eight patients. From five osteosarcoma patients $(63 \%)$ at least one cell line was obtained with a high

Table 3 Summary of sample success rates with a high degree of chromosomal abnormalities as assessed by karyotyping or FISH analysis

\begin{tabular}{lccc}
\hline WHO & Patients & Samples & Abnormal samples (\%) \\
\hline Karyotyping & 8 & 17 & $7(41)$ \\
Conventional OS & 1 & 3 & $2(67)$ \\
Ewing sarcoma & 1 & 1 & $1(100)$ \\
Myxoid LS & 10 & 21 & $10(48)$ \\
Total & & & $1(17)$ \\
FISH & 6 & 6 & $3(43)$ \\
DDLS & 7 & 7 & $4(31)$ \\
Ewing sarcoma & 13 & 13 & $14(41)$ \\
Total & 23 & 34 & \\
Total & & 6 & \\
\hline
\end{tabular}

Abbreviations: DDLS, dedifferentiated liposarcoma; FISH, fluorescence in situ hybridization; LS, liposarcoma; OS, osteosarcoma; WHO, World Health Organization. degree of abnormal karyotypes. Two samples (BX and RC) from a Ewing patient (named 'Ewing patient 1', also see below) with fast progressive and deadly disease showed a highly complex karyotype, whereas the RX derived cells were considered as normal, since they only contained a pericentric inversion of chromosome 10, which most likely represents a constitutional aberration. One cell line from a myxoid liposarcoma also had a highly complex rearranged karyotype at late passage 43. Taken together, in $48 \%$ of samples evaluated with chromosome banding analysis the majority of cells were identified as tumor cells with a tendency of higher abnormality rates at later passages (Table 3 ).

Next to karyotyping, FISH analysis was performed on six cell lines of patients with dedifferentiated liposarcoma (DDLS) and with pathologically confirmed MDM2 amplification to estimate the number of cells that exhibit MDM2 amplification (Figure 4). FISH analysis was performed after thawing passage 1 cells at passages 2-6 (median 3; 25-75\% percentile 3-4). In only one cell line (17\%) the MDM2 amplification was found in $100 \%$ of the analyzed nuclei, and in two additional samples the MDM2 amplification was found in a low percentage $(2 \%)$ of nuclei. From seven patients with Ewing sarcoma and pathologically confirmed EWSR1 rearrangements, the rearrangement was again detected in three cell lines $(43 \%)$ with high percentages ( $>90 \%$ of analyzed nuclei). Taken together, 29\% (two of seven samples; Table 3) of liposarcomas showed high degree, and two showed a low degree of abnormality. In osteosarcoma and Ewing sarcoma,

a

\begin{tabular}{|c|c|c|}
\hline Liposarcoma & & MDM2 amplification (\%) \\
\hline 1 & BX & 0 \\
\hline 2 & $\mathrm{RX}$ & 0 \\
\hline 3 & RX & 2 \\
\hline 4 & BX & 100 \\
\hline 5 & BX & 0 \\
\hline 6 & BX & 2 \\
\hline Ewing & & EWSR1 rearrangement (\%) \\
\hline 1 & BX & 100 \\
\hline 2 & BX & 0 \\
\hline 3 & BX & 0 \\
\hline 4 & BX & 90 \\
\hline 5 & BX & 0 \\
\hline 6 & BX & 99 \\
\hline 7 & $\mathbf{R X}$ & 0 \\
\hline
\end{tabular}
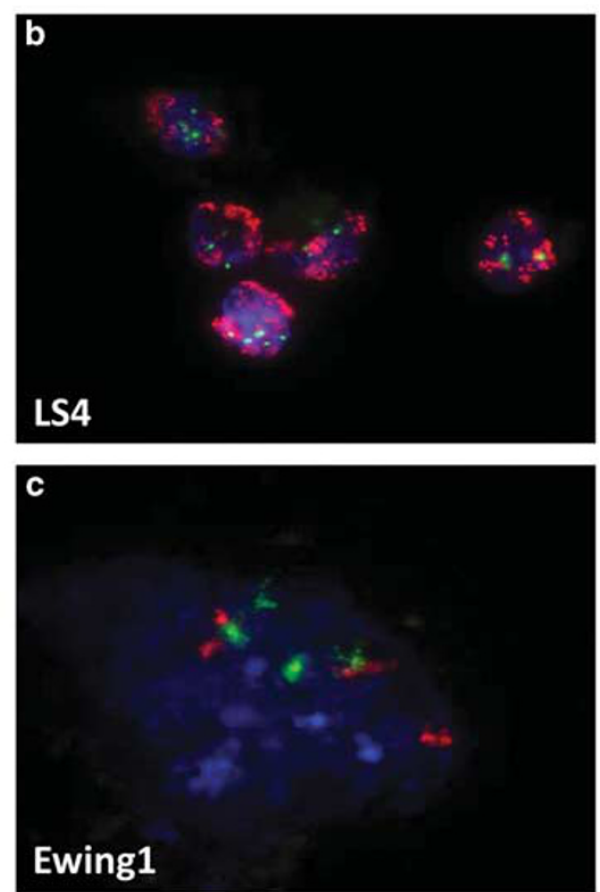

Figure 4 Fluorescence in situ hybridization analysis in cells derived from dedifferentiated liposarcoma and Ewing sarcoma. (a) Percent of abnormal cells. Representative pictures showing MDM2 amplification (b) in liposarcoma (LS) patient 4 (LS4) and rearrangement in the EWSR1 gene in Ewing patient 1 (Ewing1) (c). 
$41 \%$ ( 7 of 17 ) and $44 \%$ (4 of 9), respectively, of samples showed a high degree of abnormality. In conclusion, independent on the investigated entities and the method used for assessing the degree of abnormality, 14 (41\%) of the 34 investigated cell lines were confirmed to be composed mostly of abnormal tumor cells (Table 3).

\section{Ewing Patient 1}

From Ewing patient 1, who died 52 weeks following diagnosis, cells were obtained from naive BX (week 0), chemotherapy-treated RX (week 21) and from a recurrence (RC) at week 32. BX-derived cells showed a high degree of abnormality by karyotyping and FISH (Figures 3 and 4) and RC-derived cells were also highly abnormal by karyotyping. The short survival of this patient and the highly complex karyotype is consistent with short survival of a subgroup of Ewing patients with complex karyotypes and/or trisomy $20 .^{28}$ Therefore it was surprising to find an inversion of chromosome 10 as sole aberration in the RX-derived cells 10 (Figure 3 ). The failure to detect the highly complex karyotype seen in BX- and RC-derived cells is most likely consistent with a cytogenetic remission, as the patient underwent chemotherapy. However, it is not excluded that sarcoma cells were suppressed by non-tumor cells such as fibroblasts as often observed in long-term cultures. We therefore investigated the morphology and CD99 expression ${ }^{29}$ in these cells (Figure 5). BX-derived cells grew as loosely attached patches that did not reach confluence. RX-derived cells were firmly attached and looked fibroblast like, whereas RC-derived cells were almost unattached or became loosely attached after several days of seeding (Figure 5a). BX and RC derived strongly stained for CD99, whereas staining of RXderived cells was faint, although above isotype control (Figure 5b). Staining in suspension showed high degree of CD99-positive cells in BX- and RC-derived cells (Figure 5c). $\mathrm{RX}$-derived cells, lacking the complex karyotype, having low a

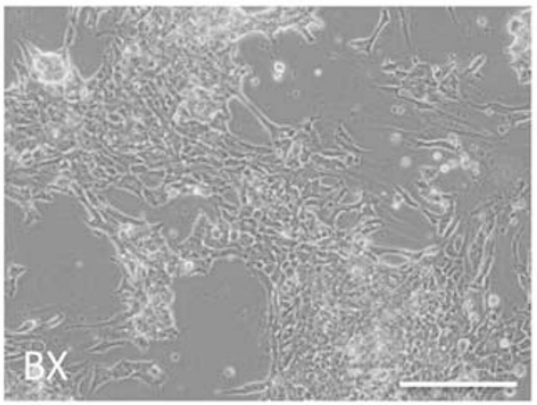

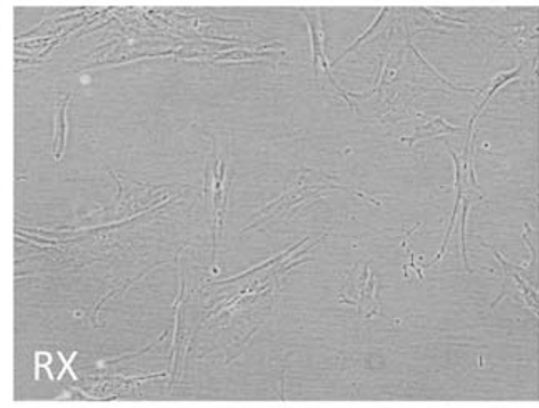

C
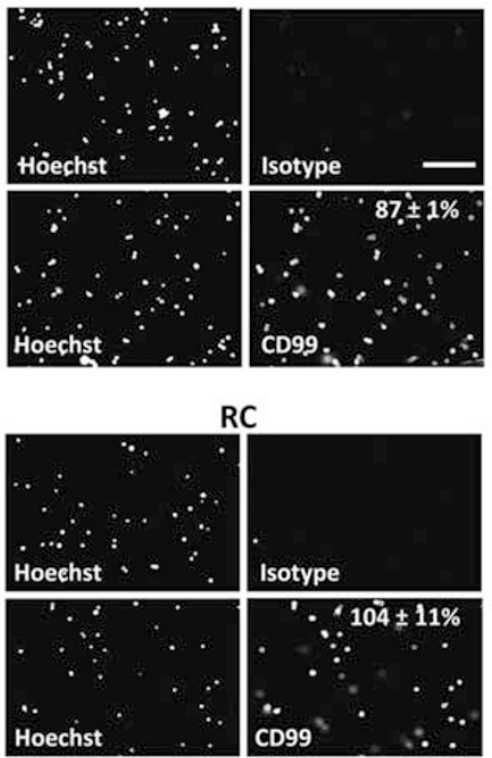

b
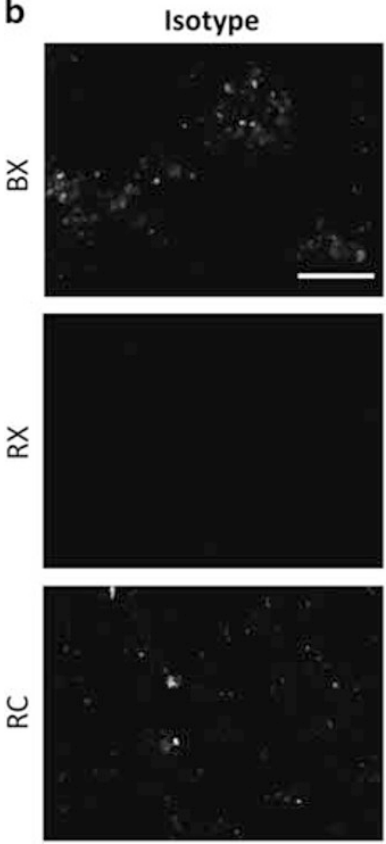

CD99
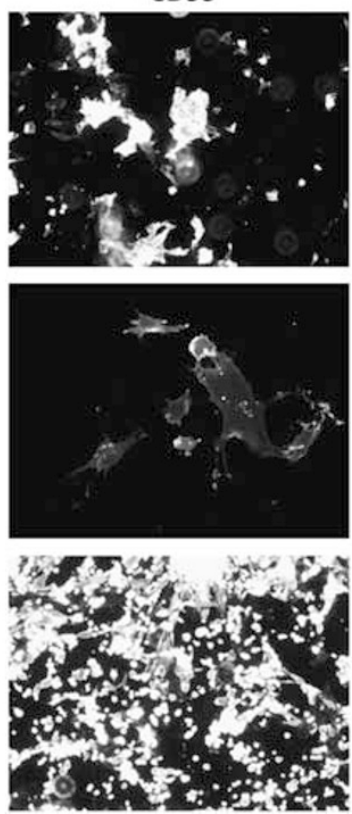

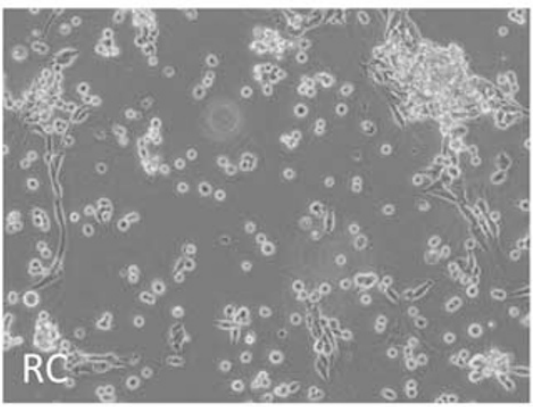

$\mathrm{BX}$

CD99

Figure 5 CD99 expression in biopsy- (BX), resection- (RX), and recurrence- (RC) derived cells from Ewing sarcoma patient 1. (a) Bright field images. (b) Staining with CD99 antibody or isotype control of cells grown on gelatin-coated glass slides. (c) Staining with CD99 antibody or isotype control and Hoechst nuclear dye of BX and RC derived cells in suspension. After staining cells were transferred to a Neubauer chamber and pictures were analyzed with ImageJ to calculate the percentage of CD99-positive cells. Scale bars, $200 \mu \mathrm{m}$. 
CD99 expression and a fibroblast-like morphology, should therefore be considered as stromal cells rather than tumor cells.

\section{DISCUSSION}

Overall, the success rates, defined as percent of samples yielding enough cells to be frozen, were high (50-100\%) in a total of 734 investigated samples, with bone sarcomas having a significant higher success rate than soft tissue sarcomas (Table 2). When low times to freezing were considered as an additional parameter for overall success, bone sarcomas had significant lower times to freezing than soft tissue sarcoma. With $75-91 \%$ success rates and times to freezing ranging from 58 to 68 days, the chondrogenic and osteogenic sarcomas and Ewing sarcoma seem the most promising entities to obtain patient-derived tumor cells, also with respect to malignancy and/or incidence. Unfortunately, the most common malignant soft tissue tumors, liposarcoma, had a low success rate and high times to freezing. Here, we speculate that the high lipid content might keep the tissues floating, thereby preventing them from adherence to the plastic dishes and ultimately cellular outgrowth.

Malignant tumor cells are expected to evade and migrate faster than less malignant ones and should therefore give rise to cells with shorter times to freezing. We therefore investigated time to freezing in those entities that include benign, intermediate, and malignant tumors. Indeed, malignant chondrogenic tumors had shorter times to freezing than benign tumors (Figure 2). Higher success rates were also related to malignancy in other tumors listed in Table $4 .^{18,30-33}$ A similar trend was observed in osteogenic tumors and tumors of uncertain differentiation. In comparison, cells derived from metastatic colon cancer could not be established by the explant technique and instead required patient-derived xenograft (PDX) transplantation, indicating an inverse relationship between success and malignancy. ${ }^{34}$ In melanoma, success rates were lower in slow cycling cells, which needed further enrichment by selective trypsinization. A slow cycling phenotype is correlated with a mesenchymal expression profile resembling de-differentiated melanoma and MAPK pathway inhibitor resistance (personal communication). ${ }^{35}$

Success rates and time to freezing may also depend on the amount of material that can be used for explantation. The amount of material is usually limited in initial BX, but the tissue is naive, whereas in RX larger amount of material is usually obtained but the material may be necrotic due to the size of the tumor and/or intervention by chemo- and/or radiotherapy. In chondrogenic tumors that usually are not treated before RX, success rates were higher and time to freezing lower than those of BX probably due to the fact that more material can be explanted from RX than BX (Figure 3). On the other hand, in adipocytic tumors that are often treated by chemo- and/or radiotherapy, success rates were lower and times to freezing higher in RX than in BX. A similar trend was observed in paired samples of the same patients from
Table 4 Success rates in different cancer entities by enzymatic digestion and/or the explant technique

\begin{tabular}{|c|c|c|c|}
\hline Tumors & Total samples & Success samples & Reference \\
\hline \multicolumn{4}{|l|}{ Enzymatic } \\
\hline Renal & 498 & $63(13 \%)$ & 30 \\
\hline Colon & 22 & $10(45 \%)$ & 33 \\
\hline \multirow[t]{2}{*}{ Hepatocellular } & 30 & $7(23 \%)$ & 31 \\
\hline & 4 & $4(100 \%)$ & 41 \\
\hline \multirow[t]{2}{*}{ Melanoma } & 16 & $2(13 \%)$ & 35 \\
\hline & $30^{a}$ & $21(70 \%)^{a}$ & 35 \\
\hline Hemangiosarcoma & 9 & $8(89 \%)$ & 18 \\
\hline Osteosarcoma & 10 & $4(40 \%)$ & 19 \\
\hline Median (25-75\%) & & $40 \%(13-89 \%)^{b}$ & \\
\hline \multicolumn{4}{|l|}{ Explant } \\
\hline Renal & 13 & $8(62 \%)$ & 42 \\
\hline \multirow[t]{4}{*}{ Colon } & 35 & $6(17 \%)$ & 43 \\
\hline & 113 & $11(10 \%)$ & 44 \\
\hline & 31 & $3(10 \%)$ & 34 \\
\hline & $19^{c}$ & $9(47 \%)^{c}$ & 34 \\
\hline Hepatocellular & $7^{c}$ & $7(100 \%)^{c}$ & 38 \\
\hline Head and neck & 79 & $9(11 \%)$ & 45 \\
\hline AMML & 13 & $4(31 \%)$ & 46 \\
\hline Pancreatic & 44 & $5(11 \%)$ & 47 \\
\hline $\mathrm{SCCL}$ & 93 & $16(17 \%)$ & 48 \\
\hline Median (25-75\%) & & $14 \%(10-28 \%)^{b}$ & \\
\hline Sarcoma & 34 & $14(41 \%)$ & This study \\
\hline
\end{tabular}

Abbreviations: AMML, acute myelomonocytic leukemia; Enz, enzymatic digest; Exp, explant; PDX, patient-derived xenograft; $\mathrm{SCCL}$, small-cell carcinoma of lung.

${ }^{a}$ After enrichment ${ }^{35}$ (absolute numbers are from personal communication by the authors).

${ }^{b}$ Without a and $c$.

'Explant after PDX.

fibrohistiocytic tumors and tumors of uncertain differentiation. This may indicate that indeed the viability of RX may be compromised, and not be compensated by higher amounts of tissue, in some sarcoma entities.

Following a biopsy or surgery, the cultivation of fresh explants may not be feasible in every hospital. Here we have shown that viable freezing of tissue in DMSO-containing medium yields a high success rate in later explants even after shipping the samples on dry ice several months after tissue withdrawal. Therefore, sharing of rare patient material between institutions and later expansion by the here described explant technique is possible.

A major issue inherent to all procedures for the isolation of cancer cells is contamination by stromal cells. We therefore estimated the amount of (abnormal) cancer cells in some 
entities known to express specific chromosomal aberrations using conventional karyotyping and/or FISH analysis for gene rearrangement or amplification. Here, we aimed to characterize the cells at early passages to avoid in vitro acquisition of genomic instability by passaging. ${ }^{3}$ Even under these stringent conditions our results for osteosarcoma closely match the $40 \%$ obtained after enzymatic digest (Table 4). ${ }^{19}$ Overall, $41 \%$ of samples in these three entities yielded cancer cells with none or minor stromal contamination. This is higher than the median of $14 \%$ in explants from several epithelial cancers listed in Table 4. Because we cannot exclude that success rates may be lower in the here not further characterized sarcoma entities, further characterization of explant-derived cells in other sarcomas is warranted. Of note, a considerable amount of samples (59\%) had an apparent normal morphology and probably are to be considered as tumor-associated stromal cells. Such stromal cells could be useful to study tumor stroma interaction in two-dimensional (2D) or three-dimensional (3D) co-culture systems in vitro ${ }^{36}$ or in vivo. ${ }^{37}$

The time to freezing was $<1$ month for $10 \%$ of the samples (not shown) and $<2$ months for $40 \%$ of samples (Figure 1). Therefore, in $57 \%$ of bone tumors and $26 \%$ of soft tissue tumors a considerable amount of cells could be obtained in $<2$ months after diagnostic biopsy (eg, for Ewing patient 1, enough BX-derived cells were obtained two months before resection). This opens the possibility to use the explant technique to assess postoperative treatment options (eg, drug screening) as described in a dog patient. ${ }^{17}$ To this end, we have shown that drug testing using patient-derived cells is feasible in 2D cultures and in 3D spheroid culture. ${ }^{38}$

There are several ways to improve further the success or to modify the here described method. First, the abnormality rates assessed here were in most cases derived from early passages, when stromal contamination is expected to be higher compared with late passages. Under the assumption that cancer cells will overgrow stromal cells that will eventually become senescent, cultures with initially low percentage of abnormality should enrich in cancer cells over time. Indeed, we found higher percentage of abnormal cells by karyotyping in later passages compared with early passages (not shown). Second, selective enrichment techniques could be applied as described (Table 4). ${ }^{35}$ Third, patient material could initially be expanded as PDX in mice that markedly increased the subsequent success in explant culture of colon cancer and hepatocellular carcinoma samples (Table 4). ${ }^{34,39}$

In conclusion, the here described explant technique proved that patient-derived cell lines from different kinds of sarcoma entities can be established for use in preclinical studies and in personalized medicine.

\section{ACKNOWLEDGMENTS}

We thank Drs Megan Aanstoos, Jackie Hartley, and Ronald Hugate from The Denver Clinic for Extremities at Risk for providing us with patient samples. Our work is supported by the University of Zurich, the Schweizerischer Verein
Balgrist (Zurich, Switzerland), the Walter L. \& Johanna Wolf Foundation (Zurich, Switzerland), the Highly Specialized Medicine for Musculoskeletal Oncology program of the Canton of Zurich, the Zurcher Krebsliga (Zurich, Switzerland), the 'Kind und Krebs' fund (Zollikerberg, Switzerland), and the Swiss National Science Foundation SNF Nr.310030_149649.

\section{DISCLOSURE/CONFLICT OF INTEREST}

The authors declare no conflict of interest.

1. Fletcher CDM, Bridge JA, Hogendoorn P et al. WHO Classification of Tumors of Soft Tissue and Bone, 4th edn. IARC Press: Lyon, France, 2013.

2. Allison DC, Carney SC, Ahlmann ER et al. A meta-analysis of osteosarcoma outcomes in the modern medical era. Sarcoma 2012;2012: 704872.

3. Muff R, Rath P, Ram Kumar RM et al. Genomic instability of osteosarcoma cell lines in culture: impact on the prediction of metastasis relevant genes. PLoS One 2015;10:e0125611.

4. Hausser HJ, Brenner RE. Phenotypic instability of Saos-2 cells in longterm culture. Biochem Biophys Res Commun 2005;333:216-222.

5. Sonobe $\mathrm{H}$, Furihata $\mathrm{M}$, Iwata $\mathrm{J}$ et al. Establishment and characterization of a new human clear-cell sarcoma cell-line, HS-MM. J Pathol 1993;169: 317-322.

6. Ikemoto S, Sugimura K, Yoshida $\mathrm{N}$ et al. Chondrosarcoma of the urinary bladder and establishment of a human chondrosarcoma cell line (OCUU-6). Hum Cell 2004;17:93-96.

7. Ohi S. Characterization, anticancer drug susceptibility and atRAinduced growth inhibition of a novel cell line (HUMEMS) established from pleural effusion of alveolar rhabdomyosarcoma of breast tissue. Hum Cell 2007;20:39-51.

8. Kudo N, Ogose A, Hotta $T$ et al. Establishment of novel human dedifferentiated chondrosarcoma cell line with osteoblastic differentiation. Virchows Arch 2007;451:691-699.

9. Nishio J, Iwasaki $\mathrm{H}$, Ishiguro $\mathrm{M}$ et al. Establishment and characterization of a novel human desmoplastic small round cell tumor cell line, JNDSRCT-1. Lab Invest 2002;82:1175-1182.

10. Kodama K, Doi O, Higashiyama $\mathrm{M}$ et al. Establishment and characterization of a new Ewing's sarcoma cell line. Cancer Genet Cytogenet 1991;57:19-30.

11. Schiavo R, Tullio C, La Grotteria M et al. Establishment and characterization of a new Ewing's sarcoma cell line from a malignant pleural effusion. Anticancer Res 2007;27:3273-3278.

12. Ariizumi $\mathrm{T}$, Ogose $\mathrm{A}$, Kawashima $\mathrm{H}$ et al. Establishment and characterization of a novel dedifferentiated liposarcoma cell line, NDDLS-1. Pathol Int 2011;61:461-468.

13. Li WW, Cordon-Cardo C, Chen Q et al. Establishment, characterization and drug sensitivity of four new human soft tissue sarcoma cell lines. Int J Cancer 1996;68:514-519.

14. Hong $\mathrm{SH}$, Kadosawa $\mathrm{T}$, Mochizuki $\mathrm{M}$ et al. Establishment and characterization of two cell lines derived from canine spontaneous osteosarcoma. J Vet Med Sci 1998;60:757-760.

15. Gillette JM, Gibbs CP, Nielsen-Preiss SM. Establishment and characterization of OS 99-1, a cell line derived from a highly aggressive primary human osteosarcoma. In Vitro Cell Dev Biol Anim 2008;44:87-95.

16. Hakozaki M, Hojo H, Sato $M$ et al. Establishment and characterization of a novel human malignant peripheral nerve sheath tumor cell line, FMS-1, that overexpresses epidermal growth factor receptor and cyclooxygenase-2. Virchows Arch 2009;455:517-526.

17. Davis LE, Hofmann NE, Li G et al. A case study of personalized therapy for osteosarcoma. Pediatr Blood Cancer 2013:60:1313-1319.

18. Fosmire SP, Dickerson EB, Scott $\mathrm{AM}$ et al. Canine malignant hemangiosarcoma as a model of primitive angiogenic endothelium. Lab Invest 2004;84:562-572.

19. Laschi M, Bernardini G, Geminiani M et al. Establishment of Four New Human Primary Cell Cultures from Chemo-Naive Italian Osteosarcoma Patients. J Cell Physiol 2015;230:2718-2727.

20. Suzuki $M$, Tominaga $N$, Ide $Y$ et al. Establishment and characterization of the rhabdomyosarcoma cell line designated NUTOS derived from the human tongue sarcoma: special reference to the susceptibility of anti-cancer drugs. Hum Cell 2010;23:65-73. 
21. Kiguchi $\mathrm{K}$, Ishiwata I, Ishiwata $\mathrm{E}$ et al. Establishment and characterization of a human liposarcoma cell line (HTLS) from the retroperitoneal liposarcoma. Hum Cell 2005;18:45-52.

22. Kawai $\mathrm{A}$, Naito $\mathrm{N}$, Yoshida $\mathrm{A}$ et al. Establishment and characterization of a biphasic synovial sarcoma cell line, SYO-1. Cancer Lett 2004;204: 105-113.

23. Rajabalian S, Hajializadeh Z, Pooraboli I et al. Establishment, characterization, and drug sensitivity of a new Ewing sarcoma cell line (SS-ES-1). J Pediatr Hematol Oncol 2010;32:e331-e337.

24. Snyder SA, Linder K, Hedan B et al. Establishment and characterization of a canine soft tissue sarcoma cell line. Vet Pathol 2011;48:482-485.

25. Azakami $D$, Shibutani $H$, Dohi $M$ et al. Establishment and characterization of canine rhabdomyosarcoma cell line CMS-C. J Vet Med Sci 2011;73:1105-1108.

26. Barch MJ, Knutsen T, Spurbeck JL. The AGT Cytogenetics Laboratory Manual. Lippincott-Raven Publishers: Philadelphia, 1997.

27. Shaffer LG, McGowan-Jordan J, Schmid M. An International System for Human Cytogenetic Nomenclature. Karger Publishers: Basel, 2013.

28. Roberts $\mathrm{P}$, Burchill SA, Brownhill $\mathrm{S}$ et al. Ploidy and karyotype complexity are powerful prognostic indicators in the Ewing's sarcoma family of tumors: a study by the United Kingdom Cancer Cytogenetics and the Children's Cancer and Leukaemia Group. Genes Chromosomes Cancer 2008;47:207-220.

29. Shibuya R, Matsuyama A, Nakamoto $M$ et al. The combination of CD99 and NKX2.2, a transcriptional target of EWSR1-FLI1, is highly specific for the diagnosis of Ewing sarcoma. Virchows Arch 2014;465:599-605.

30. Ebert $\mathrm{T}$, Bander $\mathrm{NH}$, Finstad $\mathrm{CL}$ et al. Establishment and characterization of human renal cancer and normal kidney cell lines. Cancer Res 1990;50:5531-5536.

31. Cheung PF, Yip CW, Ng LW et al. Establishment and characterization of a novel primary hepatocellular carcinoma cell line with metastatic ability in vivo. Cancer Cell Int 2014;14:103.

32. Malinin $\mathrm{TI}$, Claflin AJ, Block NL et al. Establishment of primary cell cultures from normal and neoplastic human prostate gland tissue. Prog Clin Biol Res 1980;37:161-180.

33. McBain JA, Weese JL, Meisner LF et al. Establishment and characterization of human colorectal cancer cell lines. Cancer Res 1984;44:5813-5821.

34. Dangles-Marie V, Pocard M, Richon S et al. Establishment of human colon cancer cell lines from fresh tumors versus xenografts: comparison of success rate and cell line features. Cancer Res 2007;67: 398-407.
35. Raaijmakers MI, Widmer DS, Maudrich M et al. A new live-cell biobank workflow efficiently recovers heterogeneous melanoma cells from native biopsies. Exp Dermatol 2015;24:377-380.

36. Majety M, Pradel LP, Gies M et al. Fibroblasts Influence Survival and Therapeutic Response in a 3D Co-Culture Model. PLoS One 2015;10: e0127948.

37. Han S, Delitto D, Zhang D et al. Primary outgrowth cultures are a reliable source of human pancreatic stellate cells. Lab Invest 2015;95: 1331-1340.

38. Rimann $\mathrm{M}$, Laternser $\mathrm{S}, \mathrm{Gvozdenovic} A$ et al. An in vitro osteosarcoma 3D microtissue model for drug development. J Biotechnol 2014;189: 129-135.

39. Xin $\mathrm{H}$, Wang $\mathrm{K}, \mathrm{Hu} \mathrm{G}$ et al. Establishment and characterization of 7 novel hepatocellular carcinoma cell lines from patient-derived tumor xenografts. PLoS One 2014;9:e85308.

40. Salzer-Kuntschik M, Brand G, Delling G. Determination of the degree of morphological regression following chemotherapy in malignant bone tumors. Pathologe 1983;4:135-141.

41. Pang $\mathrm{E}$, Wong $\mathrm{N}$, Lai $\mathrm{PB}$ et al. Consistent chromosome 10 rearrangements in four newly established human hepatocellular carcinoma cell lines. Genes Chromosomes Cancer 2002;33:150-159.

42. Hanak L, Slaby O, Lauerova L et al. Expression pattern of HLA class antigens in renal cell carcinoma and primary cell line cultures: methodological implications for immunotherapy. Med Sci Monit 2009;15:CR638-CR643

43. Kirkland SC, Bailey IG. Establishment and characterisation of six human colorectal adenocarcinoma cell lines. Br J Cancer 1986;53:779-785.

44. Leibovitz A, Stinson JC, McCombs WB 3rd et al. Classification of human colorectal adenocarcinoma cell lines. Cancer Res 1976;36: 4562-4569.

45. Kim SY, Chu KC, Lee HR et al. Establishment and characterization of nine new head and neck cancer cell lines. Acta Otolaryngol 1997;117: 775-784.

46. Treves AJ, Barak V, Halperin $\mathrm{M}$ et al. In vitro differentiation and establishment of cell lines derived from human myelomonocytic leukemia cells. Immunol Lett 1986;12:225-230.

47. Ruckert F, Aust D, Bohme I et al. Five primary human pancreatic adenocarcinoma cell lines established by the outgrowth method. J Surg Res 2012;172:29-39.

48. Pettengill OS, Sorenson GD, Wurster-Hill DH et al. Isolation and growth characteristics of continuous cell lines from small-cell carcinoma of the lung. Cancer 1980;45:906-918. 